by fitful naps, and had to be fed a teaspoonful at a time. Exhaustion was marked, the child alternating between apathy and fretfulness day and night. 'The limbs were moved feebly. On the second duy of this attack the temperature rose to $101^{\circ} \mathrm{F}$. 'The next day it was $100^{\circ}$; the next, nearly normal. Coincident with this there was suppression of the urine for eighteen hours, and, for a few times after, the urine stained the diapers a brownish-red. 'The day before this occurred the child had been moved into another room, as arsenic poisoning was suspected, and the sudden exacerbation of the symptoms occurred on the night of its removal. 'The papers of both rooms were then tested. 'That in the room first occupied was found to contain only a snull trace of arsenic, and was pronounced to be not a dangerous paper. The paper of the second room into which she had been moved contained a somewhat larger quantity, and was marked "perhaps dangerous." 'The child was then moved to a neighboring house, the papers of which had been recently tested and found to be free from ursenic. She at once began to improve, und continued to gain, though very gradually. After the second day she sat up and played, was bright, and took more food though not one-quarter of the regular quantity - and the lips and finger tips became pink. She contined to grow stronger, and to sleep better.

'The rooms in the house she had left were stripped of their papers, and the walls, woodwork, etc., were thoroughly washed. 'The straw-watting was swept, and wiped with damp cloths, and all hangings and cretomnes etc., of which there had been but few in the room, were removed. 'The drainage of the house, which had been found defective, was also put in proper condition, and wholly cut off from the part of the house occupied by the child, and the rooms were thoroughly aired for six days.

After having been absent for six days the child was brought back to the room which she had occupied at first, and which had undergone the treatment described above. No change in her improved condition was noted in the first twenty-four hours, but about twelve hours after this all the former symptoms returned, except the suppression of the urine. The temperature was $101^{\circ} \mathrm{F}$. 'There was no sign of gastro-intestinal disturbance, except a change in the color of the stools to a dark green. She was then taken back to the house which she had just left, and again began to improve slowly, the temperature at the end of the second day being normal On the following day she was taken to another town, involving a journey of about two hours, which she bore well. 'Three hours after her arrival, however, there was a recurrence of the collapse; the temperature was $103^{\circ} \mathrm{F}$; the bowels and stomach became distended with gas; and twelve hours later she died, apparently from heart failure.

'The autopsy was made by Dr. W. F. Whitney, who found a profound auxmia of all the organs, moderate enlargement of all the lymphatic glands of the interior of the body, and acute fatty degeneration of the heart. The liver was not fatty. No chemical analysis of the organs was made, and the urine had not been examined for arsenic at any time.

All that can be said is, that it is highly improbable that this anamia, with fatty degeneration of the heart, should have occurred except as a result of pernicious anamia, or from some toxic cause; that arsenic in toxic dose would be considered a sufficient canse; ;unl that no other was discovered, although the conditions under which the child lived were absolutely under control; finally, that the illness seemed to bear a certain relation to the room with the arsenic paper.

'The second collapse which occurred after the child's return to the room, like that in which it finally died, may very well have been purely independent of any new exposure, marking simply a stage in the progress of the disease.

Case V. 'The last case is, in brief, that of a physician of this city, who during the greater part of two months suffered from odema of the face, especially about the eyes, sometimes worse, sometimes better; and from slight pharyngitis, and a slight degree of general malaise. Almost all the wall-papers of his house were found to contain areenic, and a large trace was found in the urine. No other cause for this cedema was found, and after removal from the exposure, he quickly recovered.

The case was especially interesting because of the accuracy with which the facts could be observed, and also, because the patient, who had formerly given much attention to ornithology, using arsenic largely in curing the skins of birds, had developed a great susceptibility to the poison, so that he had been obliged to abstain from employing it altogether.

It may be added that a similar constitutional susceptibility was also said to bo present in the case of the young lady who suffered from the eruption on the face.

Neither of the last three cases were included in the list of analyses reported upon at the beginning of the paper.

\section{Elinical \$Department.}

\section{A CASE OF IMPEIRFORA'TE REC'IUM}

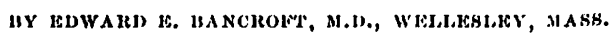

'THe pationt, an ill-nourished male infant, was two days old when first seen by me. I was called because he had failed to defecate. Superficial examination showed the anus to be normal, and tenspoonful doses of sweet oil were ordered. The next day no movement of the bowels had occurred; the nbdomen was moderately distended; the child had begun vomiting, and was in considerable pain. Further examination showed the rectum to be imperforate at a distance of about one inch from the anus.

With a nasal speculum, the child being in the lithotomy position, the imperforate diaphragm across the bowel was brought well into view, and an exploratory puncture made with an ordinary hypodermic syringe, pointing in tho direction in which the upper segment of the rectum was supposed to lie; fuces was obtained and a small bistoury was then used, making stellate incisions of reasonable extent. Very copious discharges followed.

'The artificial opening was dilated at intervals of a few days, and constipation guarded against by the daily use of sweet oil. After six or seven weeks this treatment was discontinued; and from this time on, the child has flourished (he is now seven months old) and has had normal evacuations, both in consistency and frequency.

The operation was done witl cocaine. 